\section{Die Messung von Frailty}

\section{Originalpublikation}

Dent E, Kowal P, Hoogendijk EO. Frailty measurement in research and clinical practice: a review. European journal of internal medicine 2016; 31: 3-10

\section{Einleitung}

Als Ödipus auf die Sphinx trifft, stellt diese inn vor ein Rätsel, welches sinngemäß danach fragt, was am Morgen vierfüßig, am Mittag zweifüßig, am Abend dreifüßig ist und mit steigender Anzahl der Füße an Kraft und Schnelligkeit verliert. Der Held der Sage entgegnet, dass es sich hierbei um den Menschen handle, der als Säugling auf Vieren, als Erwachsener auf Zweien und im Alter am Stock wandelt. Auch in der bildenden Kunst wird der gealterte Mensch zumeist als von Muskelschwund geplagte, vornübergebeugte Gestalt beschrieben, die sich nur mühevoll auf den Beinen halten kann und eng mit dem Tod assoziiert ist. Was hier als Synonym mit dem Altern betrachtet wird ist die Gebrechlichkeit, englisch Frailty. Jedoch ist die Frailty nicht ein Alleinstellungsmerkmal des Alters, sondern wird auch mit Krankheiten assoziiert. Gerade in einer alternden, immer inaktiveren Gesellschaft, ist es von Bedeutung, dieses wichtige Gesundheitskonstrukt in der Sport- und Bewegungstherapie zu berücksichtigen.

\section{Frailty: Definition und Modell}

Bislang existiert kein internationaler Konsens hinsichtlich einer gültigen Definition der Frailty. Relativ große Einigkeit besteht allerdings bezüglich der zentralen Charakteristika, welche die Frailty kennzeichnen. Frailty zeichnet sich durch die Dysfunktionalität mehrerer Systeme sowie einer erhöhten Empfindlichkeit gegenüber externen Stressoren aus [1]. Sowohl im Hinblick auf die Systeme als auch auf die Stressoren, handelt es sich um bio-psycho-soziale Konstrukte. Auf biologischer Seite wird beispielsweise eine kumulative Zerstörung von Zellen beschrieben, welche auf eine den gesamten Körper betreffende
Entzündungsreaktion zurückzuführen ist. Dabei ähnelt es in Entstehungsmechanismus und Symptomatik der Sarkopenie, also dem systematischen Muskel- und Funktionsverlust. Wenngleich die genauen molekularbiologischen Mechanismen noch nicht hinreichend erforscht sind, wird dennoch eine Störung der zellulären Homöostase, in Form von Ungleichgewicht zwischen den anabolen (aufbauenden) und den katabolen (abbauenden) Signalwegen der Zelle, angenommen. Verstärkt wird dieser Strukturverlust durch Mangelernährung und das Ausbleiben mechanischer Reize, sprich einem Mangel an körperlicher Aktivität. Auf psychosozialer Seite beeinflussen erhöhte Ängstlichkeit und Depressivität, soziale Isolation, Armut und ein niedriges Bildungsniveau die Wahrscheinlichkeit und Schwere der Frailty. Daneben geht Frailty mit Multimorbidität und Polypharmazie sowie mit schweren neurodegenerativen, endokrinen Erkrankungen oder Krebs einher.

Die Frailty beschreibt damit einen degenerativen Funktionszustand, der sich vom normalen Altern deutlich unterscheidet und wesentlich schneller sowie radikaler

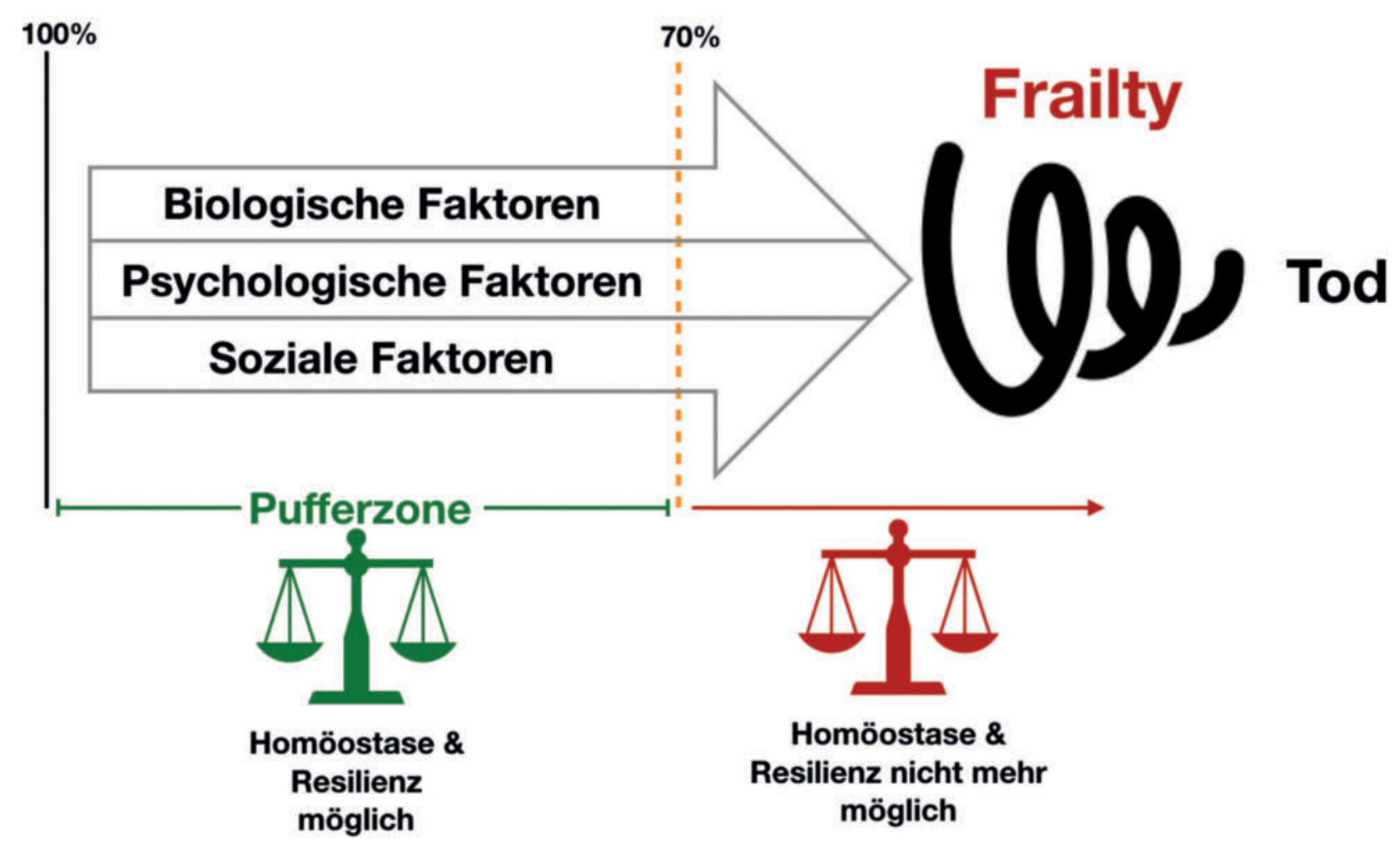

-Abb. 1 Frailty als Schwellenmodell. 
verläuft. Den Unterschied zum normalen Altern beschreiben Dent und Kollegen [2] in Anlehnung an Bortz [3] damit, dass physiologische Systeme eine Pufferzone von etwa $30 \%$ besitzen, innerhalb derer ein Funktionsverlust kompensiert werden kann und das physiologische Gleichgewicht beibehalten wird (Abb.1). Wird diese kritische Schwelle jedoch überschritten, kommt es zu einem rapiden und progressiven Funktionsverlust, der durch die körpereigenen Ressourcen nicht mehr aufgehalten werden kann. Vergleichbare Schwellenmodelle wie das Stressmodell nach Lazarus und Folkman [4] sind auch aus der Psychologie bekannt. Als Frail wird ein Mensch dann bezeichnet, wenn diese Schwelle in mehreren biologischen und psychologischen Systemen überschritten wurde, also eine progressive multisystemische und multidimensionale (bio-psycho-soziale) Degeneration beobachtet werden kann. Distal ist diese Degeneration mit einer erhöhten Mortalität assoziiert [5].

\section{Wirkung körperlicher Aktivität und körperlichen Trainings auf die Frailty}

Körperliche Inaktivität kann als einer der bedeutsamsten Risikofaktoren für die Entwicklung der Frailty gesehen werden. Dementsprechend ist ein körperlich aktiver Lebensstil eine der besten Voraussetzungen, um der körperlichen Degeneration entgegen zu wirken [7]. Aber auch bereits nach Einsetzen des fortschreitenden Muskelmasse- und Funktionsverlusts kann körperliches Training den weiteren Abbau aufhalten und ihm sogar entgegenwirken. In einer systematischen Übersichtsarbeit, in welcher 9 randomisiert kontrollierte Bewegungsstudien mit guter methodischer Qualität identifiziert wurden, konnte ein relativ konsistent positiver Effekt auf die Kraftfähigkeit, die Reduktion des Sturzrisikos, funktionale Parameter und Tests sowie die Balance und Mobilität gefunden werden [8]. Die Autoren führen jedoch gleichzeitig an, dass die Effekte aus den Studien eine enorme Variabilität aufweisen. In einem weiteren Review, welches diese Ergebnisse bestätigt [9], wird aus der existierenden
Datenlage für ein multimodales Training mit Ausdauer-, Kraft- und Koordinationskomponenten (insbesondere Balance) geworben. Um jedoch aussagekräftige Dosis-Wirkungszusammenhänge nachzuweisen und eine allgemeingültige Aussage über die Wirkung körperlichen Trainings auf die Frailty treffen zu können, müssen derlei Studienergebnisse sinnvoll synthetisiert werden. In Anbetracht der großen Heterogenität der eingesetzten Assessmentinstrumente ist dies zu diesem Zeitpunkt jedoch noch nicht möglich.

\section{Erfassung der Frailty}

Erste Anstrengungen zur Erfassung der Frailty wurden in den 1990er-Jahren unternommen. Hier wurden erste Kombinationscores etabliert, in die physiologische wie motorische Parameter einflossen. Seitdem ist das zur Verfügung stehende Inventar an Testbatterien gewachsen, ohne dass jedoch ein Goldstandard etabliert werden konnte. Um einen solchen zu schaffen, fordern Dent und Kollegen [2], dass die Assessmentinstrumente folgenden Gütekriterien genügen:

1. Konstruktvalidität: Die Instrumente sollen die Frailty valide erfassen, d. h. in ihrer Multidimensionalität.

2. Prädiktive Validität: Die Instrumente sollen wichtige klinische Outcomes zuverlässig vorhersagen können.

3. Reliabilität in der Abbildung von Interventionseffekten: Die Instrumente sollen veränderungssensitiv auf Interventionen reagieren.

4. Theoriegetrieben: Den Instrumenten soll eine fundierte biologische Theorie zugrunde liegen.

5. Anwendbarkeit: Die Instrumente sollen sowohl für den wissenschaftlichen als auch den klinischen Kontext gut anwendbar sein. Hier spielt natürlich auch das Nebengütekriterium der Testökonomie, also der Aufwand und die Kosten, die mit der Messung verbunden sind, eine Rolle.

6. In ihrem systematischen Review konnten Dent und Kollegen [2] in 422 Studien 29 verschiedene Messinstrumente zur Operationalisierung der Frailty identifizieren, wovon im Folgenden 6 Instrumente vorgestellt werden, die ihre Konstruktvalidität über verschiedene Kollektive bestätigen konnten.

\section{Frailty Indizes (FI)}

Die in den Studien am häufigsten anzutreffenden Assessmentinstrumente sind die FI. Hierbei wird der FI an die entsprechende Kohorte angepasst. Die vorausgehende Itemselektion folgt hierbei klar definierten Kriterien [10], wie z.B. einer eindeutigen Gesundheitseinschränkung, einer positiven Korrelation mit dem kalendarischen Alter, ausreichender Heterogenität (keine Merkmale, die ab einem bestimmten Alter bei fast jedem auftreten wie Altersweitsichtigkeit) und dass die Items verschiedene Systeme überspannen müssen (nicht nur z.B. die Kognition). Wurde ein hinreichend umfassendes Itemuniversum geschaffen, wird dieses als Referenz herangezogen. Der Fl entspricht hierbei dem Anteil der Einschränkungen des gesamten Itemuniversums, unter denen ein Mensch leidet. D.h. $0=$ keine Einschränkungen und 1=alle Einschränkungen. Dabei erfolgt keine Gewichtung der einzelnen Items, d.h. allen wird dieselbe Wichtigkeit und dieselbe Aussagekraft hinsichtlich der Frailty beigemessen. Der Literatur ist ein Grenzwert von etwa 2/3 zu entnehmen, d.h. dass jenseits dieser Schwelle ein ferneres Überleben eher unwahrscheinlich ist $[10,11]$. Zu den FI liegen bereits einige Validierungsstudien vor, und sie erfüllen die geforderte prädiktive Validität, d. h. sie zeigen eine starke Korrelation mit negativen klinischen Kriterien. Hierin übertreffen sie auch andere Frailty Assessmentinstrumente.

\section{Study of Osteoporotic Fractures (SOF) frailty index}

Der SOF ist ein extrem sparsamer und einfacher Index, bestehend aus lediglich drei Items:

- Nicht intentionaler Gewichtsverlust von $>5 \%$ binnen des letzten Jahres

- Verneinung der Frage „Fühlen Sie sich energiegeladen?“ (,Do you feel full of energy?")

- Unfähigkeit, 5-mal vom Stuhl aufzustehen und sich wieder hinzusetzen (Chair-Rise)

Die Klassifizierung als Frail tritt dann ein, wenn mindestens 2 der 3 obigen Kriterien 
erfüllt werden. Hierdurch kann es unter medizinischen Akutbedingungen zu systematischen Überschätzungen der Frailtyprävalenz kommen. Dennoch hat sich der SOF als valides Untersuchungsinstrument behaupten und die konvergente Validität zu den umfangreicheren $\mathrm{FI}$ bestätigen können.

\section{Edmonton Frailty Scale (EFS)}

Der EFS umfasst 17 Items, die sich auf 9 Dimensionen verteilen (Kognition, allgemeiner Gesundheitsstatus, subjektive Gesundheitswahrnehmung, Autonomie, soziale Unterstützung, Polypharmazie, Stimmung, Kontinenz, Funktionsfähigkeit). Anhand der Anzahl positiver Items wird der Grad der Frailty bestimmt und in 6 Kategorien eingeteilt: Nicht Frail (0-5), Anfällig (6-7), leichte Frailty (8-9), Moderate Frailty (1011) und ausgeprägte Frailty (12-17). Durch die 9 Dimensionen ist der EFS relativ einfach und doch hinreichend komplex, um der Multidimensionalität der Frailty gerecht zu werden, und wird daher immer häufiger im klinischen Kontext eingesetzt.

\section{Fatigue, Resistance, Ambulation, Illness, Loss of Weight (FRAIL) Index}

Der FRAIL besteht aus 5 Komponenten: Fatigue, Schwierigkeiten beim Treppensteigen, langsame Ganggeschwindigkeit, Multimorbidität und Gewichtsverlust (>5 \% binnen des letzten Jahres) [12]. Treffen mehr als drei dieser Komponenten zu, erfolgt die Klassifikation als Frail. Der Test basiert auf der Selbsteinschätzung der Patienten und nicht auf objektiven Tests, wodurch sich der Test sehr einfach ausführen lässt. Hierbei fehlen allerdings noch Validierungsergebnisse außerhalb des klinischen Settings.

\section{Multidimensional Prognostic Instrument (MPI)}

Der MPI besteht aus 8 Dimensionen: Polypharmazie, instrumentelle Alltagsaktivitäten, Alltagsaktivitäten, Kognition, Ernährung, Risiko, Druckstellen zu entwickeln, Komorbiditäten und Lebensumstände. Hierbei werden jeder der 8 Dimensionen mehrere Items zugeordnet, z. B. im Fall der instrumentellen Alltagsaktivitäten: Finanzen organisieren, Einnahme von Medikamenten, Telefon nutzen, Einkaufen, Nutzung von Verkehrsmitteln,
Vorbereiten von Mahlzeiten, Bewerkstelligen des Haushalts und Wäschewaschen [13]. Jeder Dimension werden 0 Punkte, sollten keine Einschränkungen vorliegen, 0,5 Punkte, sollten kleinere Einschränkungen und 1 Punkt, sollten größere Einschränkungen vorliegen, zugeordnet. Anschließend wird der Mittelwert aus den 8 Bepunktungen berechnet, welcher dann als Frailty Index betrachtet wird. Die Klassifikation als Frail erfolgt bei einem Score von $>0.66$. Erste Validierungsergebnisse sind vielversprechend und zeigen, dass der MPI anderen Instrumenten überlegen ist.

\section{Tilburg Frailty Indicator (TFI)}

Der TFI besteht aus 15 Items, welche der Patient selbst beantwortet. Diese werden 3 Dimensionen (physisch, psychisch, sozial) zugeordnet. In der physischen Dimension wird nach dem subjektiven Gesundheitszustand, dem Gewichtsverlust, den Gangbeschwerden, den Balancebeschwerden, den Hör- und Seheinschränkungen sowie nach den Einschränkungen der Griffkraft und Müdigkeit gefragt. Die psychologische Dimension wird durch Kognition, Depressivität, Ängstlichkeit und Kompensationsstrategien gebildet. Als soziale Indikatoren fragt die Testbatterie danach, ob man allein lebt, sozial isoliert ist und soziale Unterstützung erhält. Liegen in mehr als 5 Items Einschränkungen vor, erfolgt die Klassifikation als Frail. Die Testbatterie demonstriert eine gute Validität und Reliabilität.

\section{Motorische Tests}

Bis hier handelte es sich bei allen Items um Fremd- oder Selbstbewertungen, oder es wurde sich der medical records der Patienten bedient. Als wichtige motorische Indikatoren der Frailty wird von den Autoren der Übersichtsarbeit insbesondere die Ganggeschwindigkeit und die Griffkraft angeführt. Insbesondere der Ganggeschwindigkeit kommt hierbei eine herausragende Stellung bei: „Gait speed [...] in all likelihood, may be best indicator of frailty in Fried's Frailty Index. (S.7).“ [2] (Der Fried's Frailty Index ist hierbei der umfangreichste, also der am meisten Items umfassende FI). Diese Bedeutung wird durch die enge Assoziation mit Gesundheitsbeeinträchtigungen begründet. Allerdings besitzt die Ganggeschwindigkeit eine niedrige Spezifität, d.h. sie führt zu einer Überschätzung der Frailtyprävalenz.

\section{Praktische Implikation und Konklusion}

Frailty ist ein multidimensionales, biopsycho-soziales Konstrukt, welches eng mit dem Alter und degenerativen Erkrankungen assoziiert ist sowie eine hohe Vorhersagekraft auf die Sterbewahrscheinlichkeit besitzt. Für die Konzeption der Sport- und Bewegungstherapie kann der Fokus auf die Frailty daher von enormer Bedeutung sein. Um eine sinnvolle Planung sowie eine Sicherung der Ergebnisqualität zu gewährleisten, bedarf es jedoch valider und testökonomischer Assessmentinstrumente. Leider liegt bislang kein Goldstandard zur Frailtymessung vor. Stattdessen wird der Therapeut mit einer Vielzahl an Messinstrumenten konfrontiert, welche die Literatur bietet. Die in der vorgestellten Übersichtsarbeit präsentierten Instrumente zielen hierbei vor allem auf ein medizinisches Publikum ab, d.h. sie wurden an für den Arzt relevanten, klinischen Kriterien validiert. So berichten die Autoren im Kontext des TFI, dass die funktionalen Items im Hinblick auf klinisch relevante Ereignisse prädiktive Validität besitzen, die sozialen Items hierbei jedoch versagen. Diese sozialen Items könnten aus einer stärker bio-psycho-sozialen oder patientenorientierten Perspektive allerdings von großer Bedeutung sein-um den Jahren mehr Leben statt dem Leben lediglich mehr Jahre zu geben. Auch ungewiss ist, inwieweit die häufig nur dichotomen Skalen (Ja/Nein) hinreichend sensitiv sind, um einen Therapieerfolg abzubilden. Es könnte ja auch sein, dass bereits kleine bis moderate Veränderungen, welche dafür über mehrere Dimensionen hinweg beobachtet werden, in der Summe ebenso wichtig sind. Das Konstrukt der Frailty sollte in die sport- und bewegungstherapeutische Praxis unbedingt stärkeren Einzug finden. Ebenso wie Assessmentinstrumente und Testbatterien unter sport- und bewegungstherapeutischen 
Gesichtspunkten definiert und genutzt werden sollten, um ein zielorientiertes und patientenzentriertes Arbeiten zu gewährleisten.

Literatur

[1] Clegg A, Young J, lliffe $S$ et al. Frailty in elderly people. The lancet 2013; 381: $752-$ 762

[2] Dent E, Kowal P, Hoogendijk EO. Frailty measurement in research and clinical practice: a review. European journal of internal medicine 2016; 31: 3-10

[3] Bortz W. Human aging: normal and abnormal. Geriatric medicine Cambridge: Blackwell Scientific Publisher 1996

[4] Lazarus RS, Folkman S. Stress, appraisal, and coping. 9. [print.]. Aufl. New York: Springer; 1996

[5] Lin J-R, Kao EH-C, Weng S-C et al. A Study of Frailty, Mortality, and Health Depreciation Factors in Older Adults. International Journal of Environmental Research and Public Health 2020; $17: 211$

[6] Viña J, Rodriguez-Mañas L, Salvador-Pascual A et al. Exercise: the lifelong supplement for healthy ageing and slowing down the onset of frailty. The Journal of physiology 2016; 594: 1989-1999

[7] De Labra C, Guimaraes-Pinheiro C, Maseda A et al. Effects of physical exercise interventions in frail older adults: a systematic review of randomized controlled trials. BMC Geriatrics 2015; 15: 154

[8] Marzetti E, Calvani R, Tosato M et al. Physical activity and exercise as countermeasures to physical frailty and sarcopenia. Aging clinical and experimental research 2017; 29: 35-42

[9] Searle SD, Mitnitski A, Gahbauer EA et al. A standard procedure for creating a frailty index. BMC Geriatrics 2008; 8: 24

[10] Rockwood K, Mitnitski A. Limits to deficit accumulation in elderly people. Mechanisms of ageing and development 2006; 127: 494-496

[11] Morley JE, Malmstrom T, Miller D. A simple frailty questionnaire (FRAIL) predicts outcomes in middle aged African Americans. The journal of nutrition, health \& aging 2012; 601-608

[12] Pilotto A, Ferrucci L, Franceschi $M$ et al. Development and validation of a multidimensional prognostic index for one-year mortality from comprehensive geriatric assessment in hospi-talized older patients. Rejuvenation research 2008;11: 151-161

\section{Korrespondenzadresse}

Maximilian Köppel

Deutscher Verband für Gesundheitssport und Sporttherapie e. V.

Vogelsanger Weg 48

50354 Hürth-Efferen

Deutschland

maximilian.koeppel@outlook.de 